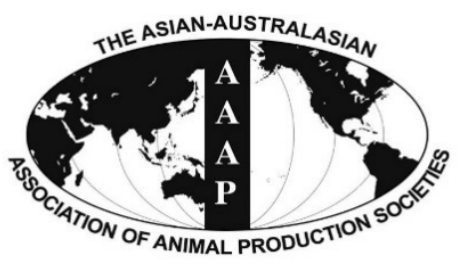

\title{
Effects of Extracellular Matrix Protein-derived Signaling on the Maintenance of the Undifferentiated State of Spermatogonial Stem Cells from Porcine Neonatal Testis
}

\author{
Min Hee Park ${ }^{1}$, Ji Eun Park ${ }^{1}$, Min Seong Kim ${ }^{1}$, Kwon Young Lee ${ }^{2}$, Jae Yeon Hwang ${ }^{3}$, Jung Im Yun ${ }^{4}$, \\ Jung Hoon Choi ${ }^{2}$, Eunsong Lee ${ }^{2}$, and Seung Tae Lee ${ }^{1,3, *}$ \\ ${ }^{1}$ Department of Animal Life Science, Kangwon National University, Chuncheon 200-701, Korea
}

\begin{abstract}
In general, the seminiferous tubule basement membrane (STBM), comprising laminin, collagen IV, perlecan, and entactin, plays an important role in self-renewal and spermatogenesis of spermatogonial stem cells (SSCs) in the testis. However, among the diverse extracellular matrix (ECM) proteins constituting the STBM, the mechanism by which each regulates SSC fate has yet to be revealed. Accordingly, we investigated the effects of various ECM proteins on the maintenance of the undifferentiated state of SSCs in pigs. First, an extracellular signaling-free culture system was optimized, and alkaline phosphatase (AP) activity and transcriptional regulation of SSC-specific genes were analyzed in porcine SSCs (pSSCs) cultured for 1, 3, and 5 days on non-, laminin- and collagen IV-coated Petri dishes in the optimized culture system. The microenvironment consisting of glial cell-derived neurotrophic factor (GDNF)-supplemented mouse embryonic stem cell culture medium (mESCCM) (GDNF-mESCCM) demonstrated the highest efficiency in the maintenance of AP activity. Moreover, under the established extracellular signaling-free microenvironment, effective maintenance of AP activity and SSC-specific gene expression was detected in pSSCs experiencing laminin-derived signaling. From these results, we believe that laminin can serve as an extracellular niche factor required for the in vitro maintenance of undifferentiated pSSCs in the establishment of the pSSC culture system. (Key Words: Porcine, Spermatogonial Stem Cells, Undifferentiation, Extracellular Matrix Proteins)
\end{abstract}

\section{INTRODUCTION}

During male embryogenesis, primordial germ cells (PGCs) derived from the epiblast relocate in the genital ridges and experience differentiation into gonocytes (McLaren, 2003). Subsequently, gonocytes are located in the basement membrane of seminiferous tubule and

\footnotetext{
* Corresponding Author: Seung Tae Lee. Tel: +82-33-250-8638, Fax: +82-33-251-7719, E-mail: stlee76@kangwon.ac.kr

${ }^{2}$ College of Veterinary Medicine and Institute of Veterinary Science, Kangwon National University, Chuncheon 200-701, Korea.

${ }^{3}$ Division of Applied Animal Science, Kangwon National University, Chuncheon 200-701, Korea.

4 Division of Animal Resource Science, Kangwon National University, Chuncheon 200-701, Korea.

Submitted Oct. 20, 2015; Revised Dec. 17, 2015; Accepted Jan. 25, 2016
}

differentiate into spermatogonial stem cells ( $\mathrm{SSCs}$ ) (de Rooij and Grootegoed, 1998; Brinster, 2002).

SSCs are male germline stem cells with indefinite selfrenewal ability (Hofmann, 2008; Luo et al., 2009; KanatsuShinohara and Shinohara, 2013) and are unipotent with respect to their differentiating into sperm through spermatogenesis in seminiferous tubule in the testis (Aponte et al., 2005; Luo et al., 2009; Lee et al., 2013). Simultaneously, SSCs can acquire pluripotency differentiating into three germ-layer cells by external specific stimulation (Guan et al., 2006; Simon et al., 2010; Lim et al., 2013) and produce transgenic sperms by introducing target genes into cells (Nagano et al., 2001; Lavitrano et al., 2006; de Barros et al., 2012). Accordingly, they serve as a useful tool for understanding spermatogenesis (Oatley and Brinster, 2008; Phillips et al., 2010; Kanatsu-Shinohara and Shinohara, 2013), preserving 
male reproduction (Phillips et al., 2010), overcoming male infertility (Kubota and Brinster, 2006; Vlajković et al., 2012), conducting patient-specific cell therapy (Daley and Scadden, 2008; Vlajković et al., 2012), and producing transgenic animals (de Barros et al., 2012).

SSCs are located on the seminiferous tubule basement membrane (STBM) of the testis (Brinster, 2002; Oatley and Brinster, 2006; Hofmann, 2008). The STBM comprises extracellular matrix (ECM) proteins, such as laminin, collagen IV, perlecan, and entactin (Yazama et al., 1997; Siu and Cheng, 2004). Among them, laminin consisting of $\alpha, \beta$, and $\gamma$ chains as a heterotrimer (Ooba et al., 2008) play an important role in constructing STBM by constituting networks independently (Ooba et al., 2008) or with collagen IV via nidogen and perlecan (Ooba et al., 2008). Simultaneously, diverse integrin heterodimer or membrane protein binding sites embedded in laminin can induce specific cellular responses by regulating intracellular signal pathway (Timpl et al., 1979; Ooba et al., 2008; Chen et al., 2013; Gattazzo et al., 2014). Also, collagen IV with triple helix structure enhance structural stability of STBM (Pöschl et al., 2004; Khoshnoodi et al., 2008) and influence on cell adhesion (Aumailley and Timpl, 1986; Khoshnoodi et al., 2008), migration (Khoshnoodi et al., 2008; Öhlund et al., 2013) and differentiation (Khoshnoodi et al., 2008; Gattazzo et al., 2014). Furthermore, previous studies have revealed that relocation of SSCs into the STBM to the apical lumen induces differentiation into sperm (Siu and Cheng, 2008), indicating that ECM proteins constituting the STBM play an important role in SSC fate. However, few studies have investigated the effects of each ECM protein on SSC properties in all species.

In the study described herein, we attempted to evaluate how different ECM protein-derived signals regulate the undifferentiated state of SSCs in pig, which is regarded as a useful species for studying xenotransplantation, disease models, etc. Alkaline phosphatase (AP) activity and transcriptional levels of SSC-specific genes were measured and compared among other SSCs experiencing each ECM protein-derived signal.

\section{MATERIALS AND METHODS}

\section{Animals}

Testes derived from purebred (Yorkshire $\times$ Yorkshire) or crossbred (Landrace $\times$ Yorkshire) male neonatal pigs (from 1- to 4-day-old), generously supplied from Gumbo Inc. (Wonju, Korea), were collected through routine castration surgery. All experimental animal procedures were permitted by Institutional Animal Care and Use Committee (IACUC) of Kangwon National University (IACUC approval No. KW-131106-1). Moreover, the procedures were performed in accordance with the Animal Care and Use Guideline of
Kangwon National University.

\section{Isolation of testicular cells from porcine testes}

Transportation of testes from a local farm (Gumbo Inc., Korea) to our laboratory was conducted in ice-cold Dulbecco's phosphate-buffered saline (DPBS; Welgene Inc., Daegu, Korea) supplemented with $1 \%(\mathrm{v} / \mathrm{v})$ antibioticantimycotic solution (Welgene, Korea) within $1 \mathrm{~h}$. The tunica albuginea and epididymis of testis were removed and the seminiferous tubules were digested by $0.1 \%(\mathrm{w} / \mathrm{v})$ type IV collagenase (Worthington Biochemical, Lakewood, NJ, USA) in high glucose Dulbecco's modified Eagle's medium (DMEM; Welgene, Korea) at $37^{\circ} \mathrm{C}$ for $15 \mathrm{~min}$. Subsequently, the fragments of the seminiferous tubules were dissociated by $0.1 \%(\mathrm{w} / \mathrm{v})$ hyaluronidase (SigmaAldrich, St. Louis, MO, USA) in high glucose DMEM at $37^{\circ} \mathrm{C}$ for $10 \mathrm{~min}$ and the dissociated fragment of the seminiferous tubule were promptly digested by $0.25 \%$ trypsin-ethylenediaminetetraacetic acid (Welgene, Korea) at $37^{\circ} \mathrm{C}$ for $10 \mathrm{~min}$. After washing the dissociated testicular cells in DMEM supplemented with $10 \%(\mathrm{v} / \mathrm{v})$ heatinactivated fetal bovine serum (FBS; HyClone, Logan, UT, USA), they were filtered using a 70- $\mu$ m nylon strainer (SPL, Pocheon, Korea) for eliminating myoid and Sertoli cells. Moreover, erythrocytes in the cells were removed by red blood cell lysis buffer (Sigma-Aldrich, USA) for $15 \mathrm{~min}$ at room temperature.

\section{Collection of pSSCs from testicular cells}

To sort pSSCs from the testicular cells was conducted by Petri dish plating post-differential plating method described previously (Park et al., 2014). Briefly, $5 \times 10^{6}$ testicular cells re-suspended in high glucose DMEM supplemented with $10 \%$ (v/v) heat-inactivated FBS and 1\% (v/v) antibiotic-antimycotic solution were seeded on 100$\mathrm{mm}$ Petri dish (SPL, Korea) coated with $0.1 \%(\mathrm{w} / \mathrm{v})$ gelatin (Sigma-Aldrich, USA) and incubated for $16 \mathrm{~h}$ at $37^{\circ} \mathrm{C}$. Subsequently, $1 \times 10^{6}$ of pSSCs suspended in medium were plated on $35-\mathrm{mm}$ Petri dish and incubated overnight. The suspended pSSCs were collected and enumerated using a hemocytometer.

\section{Preparation of ECM protein-coated Petri dishes}

To obtain laminin (Sigma-Aldrich, USA)-coated culture dishes, $2 \mathrm{~mL}$ of $40 \mu \mathrm{g} / \mathrm{mL}$ laminin solution were spread onto $35-\mathrm{mm}$ Petri dishes and incubated for $2 \mathrm{~h}$ at $37^{\circ} \mathrm{C}$. Laminin-coated plates were rinsed three times with DPBS. To obtain collagen IV (Sigma-Aldrich, USA)-coated culture dishes, $2 \mathrm{~mL}$ of $8 \mu \mathrm{g} / \mathrm{mL}$ collagen IV solution were spread onto 35-mm Petri dishes and incubated overnight at $4^{\circ} \mathrm{C}$ without air drying. Collagen IV-coated plates were then sterilized by exposure to ultraviolet light for $1 \mathrm{~h}$. 


\section{Culture of pSSCs and experimental design}

To identify culture medium supporting the maintenance of AP activity in the suspension culture, isolated pSSCs on non-coated 35-mm Petri dishes were cultured for 6 days in glial cell-derived neurotrophic factor (GDNF)supplemented mouse embryonic stem cell culture medium (mESCCM) (GDNF-mESCCM) and porcine spermatogonial stem cell culture medium (pSSCCM) at $37^{\circ} \mathrm{C}$ under $5 \%$ $\mathrm{CO}_{2}$ in a humidified air atmosphere. AP activity was investigated daily. Subsequently, for the purpose of investigating the effects of ECM protein-derived signaling on pSSC undifferentiation, isolated pSSCs were cultured for 5 days on non-, laminin- and collagen IV-coated 35-mm Petri dishes, and AP activity and SSC-specific gene expression were analyzed in pSSCs cultured for $0,1,3$, and 5 days.

\section{Culture medium}

GDNF-mESCCM consisted of high-glucose DMEM supplemented with $15 \%(\mathrm{v} / \mathrm{v})$ heat-inactivated FBS, 0.1 $\mathrm{mM} \beta$-mercaptoethanol (Gibco Invitrogen, Grand Island, NY, USA), $1 \%(\mathrm{v} / \mathrm{v})$ non-essential amino acid (NEAA; Gibco Invitrogen, USA), $2 \mathrm{mM}$ L-glutamine (Gibco Invitrogen, USA), 1\% (v/v) antibiotic-antimycotic solution, $1,000 \mathrm{U} / \mathrm{mL}$ mouse leukemia inhibitory factor (mLIF; Chemicon International, Inc., Temecula, CA, USA), and 10 ng/mL GDNF (R\&D Systems, Inc., Minneapolis, MN, USA). MEM alpha medium (Gibco Invitrogen, USA), which served as pSSCCM, was supplemented with $1 \%(\mathrm{v} / \mathrm{v})$ antibiotic-antimycotic solution, $1 \%(\mathrm{v} / \mathrm{v})$ NEAA, $0.1 \mathrm{mM}$ $\beta$-mercaptoethanol, N2-1 supplement (Merck Millipore; Darmstadt, Germany), DL-lactic acid (Sigma-Aldrich, USA), $1 \%$ (v/v) MEM vitamin solution (Sigma-Aldrich, USA), $30 \mathrm{ng} / \mathrm{mL} \beta$-estradiol (Sigma-Aldrich, USA), 1\% $(\mathrm{v} / \mathrm{v})$ heat-inactivated FBS, $10 \mathrm{ng} / \mathrm{mL}$ basic fibroblast growth factor (bFGF; PeproTech, Inc., Rocky Hill, NJ, USA), $20 \mathrm{ng} / \mathrm{mL}$ epidermal growth factor (EGF; PeproTech, Inc., USA), 1,000 U/mL mLIF, and $10 \mathrm{ng} / \mathrm{mL}$ GDNF.

\section{Alkaline phosphatase staining}

Cells fixed with $4 \%(\mathrm{v} / \mathrm{v})$ paraformaldehyde (Junsei
Chemical Co., Ltd., Chuo-ku, Japan) for $10 \mathrm{~min}$ at room temperature were washed twice with DPBS. The fixed cells were stained with AP staining solution consisting of $0.1 \mathrm{M}$ Tris buffer ( $\mathrm{pH} 8.2$ ) supplemented with $0.2 \mathrm{mg} / \mathrm{mL}$ naphthol AS-MX phosphate (Sigma-Aldrich, USA), 2\% (v/v) dimethyl formamide (Sigma-Aldrich, USA), and $1 \mathrm{mg} / \mathrm{mL}$ Fast Red TR salt (Sigma-Aldrich, USA) for $90 \mathrm{~min}$ at room temperature. Subsequently, the wells were rinsed twice with DPBS and then the percentage of the AP-positive cells was counted by a hemocytometer.

\section{Quantitative real-time polymerase chain reaction}

According to the manufacturer's instructions, total mRNA was extracted from the cells by the Dynabeads mRNA Direct Kit (Ambion, Austin, TX, USA) and cDNA synthesis was conducted using the ReverTra Ace qPCR RT Master Mix with gDNA remover kit (Toyobo, Osaka, Japan). Subsequently, quantification of the specific gene expression was performed with a real-time polymerase chain reaction (PCR) Master Mix (Toyobo, Japan) under the 7500 Real time PCR system (Applied Biosystems, Foster City, CA, USA). Melting curve data was analyzed for identifying PCR specificity and normalization of the specific gene expression was performed by comparison to the mRNA level of glyceraldehyde-3-phosphate dehydrogenase $(G A P D H)$. Comparative transcriptional level was presented as $2^{-\Delta \Delta \mathrm{Ct}}$, where $\mathrm{Ct}=$ threshold cycle for target amplification, $\Delta \mathrm{Ct}=\mathrm{Ct}_{\text {target gene }}$ (specific genes for each sample) $-\mathrm{Ct}_{\text {internal reference }}(G A P D H$ for each sample), and $\Delta \Delta \mathrm{Ct}=\Delta \mathrm{Ct}_{\text {sample }}$ (treatment sample of each experiment) - $\Delta \mathrm{Ct}_{\text {calibrator }}$ (control sample in each experiment). General information and sequences of primers were designed by Primer3 software (Whitehead Institute/MIT Center for Genome Research) with information of cDNA sequences obtained from GenBank for pig and described in Table 1.

\section{Statistical analysis}

The statistical analysis of all the numerical data shown in each experiment was performed by the Statistical Analysis System (SAS) program. Furthermore, the results were compared by the least-square or DUNCAN method,

Table 1. Oligonucleotide primers and PCR cycling conditions

\begin{tabular}{|c|c|c|c|c|c|}
\hline \multirow{2}{*}{ Genes } & \multirow{2}{*}{ GenBank number } & \multicolumn{2}{|c|}{ Primer sequence } & \multirow{2}{*}{ Size (bp) } & \multirow{2}{*}{ Temp $\left({ }^{\circ} \mathrm{C}\right)$} \\
\hline & & Sense $\left(5^{\prime}>3^{\prime}\right)$ & Anti-sense $\left(5^{\prime}>3^{\prime}\right)$ & & \\
\hline GAPDH & NM_001206359.1 & AGGGCTGCTTTTAACTCTGGCAA & GATGGTGATGGCCTTTCCATTG & 180 & 60 \\
\hline OCT4 & NM_001113060.1 & CGCGAAGCTGGACAAGGAGA & CAAAGTGAGCCCCACATCGG & 151 & 60 \\
\hline NANOG & NM_001129971.1 & AACCAAACCTGGAACAGCCAGAC & GTTTCCAAGACGGCCTCCAAAT & 158 & 60 \\
\hline EPCAM & NM_214419.1 & CAATGCAGGGTCTACAGGCTGG & TGCATCTCGCCCATCTCCTTT & 154 & 60 \\
\hline THY1 & NM_001146129.1 & GTGCTCTTGGGCACTGTGGG & TCTTGCTGGAGATGCTGGGC & 178 & 60 \\
\hline UCHL1 & NM 213763.2 & TCCGGAAGACAGAGCAAAATGC & TAGAGGTGGCCATCCACGTTGT & 150 & 60 \\
\hline
\end{tabular}

PCR, polymerase chain reaction; Temp, temperature; GAPDH, glyceraldehyde-3-phosphate dehydrogenase; OCT4, octamer-binding transcription factor 4; NANOG, homeobox transcription factor nanog; EPCAM, epithelial cellular adhesion molecule; THY1, THY-1 T-cell antigen; UCHL1, ubiquitin carboxyl-terminal esterase L1. 
when a significance of the principal effects through variance (analysis of variance) analysis in the SAS package was detected. $p$ values less than 0.05 were regarded as indicative of significant differences.

\section{RESULTS}

Establishment of the extracellular signaling-free microenvironment

Firstly, in order to evaluate the precise effects of ECM protein-derived signaling on the maintenance of pSSC undifferentiation, microenvironments that did not provide pSSCs with extracellular signals were constructed. Isolated pSSCs were plated onto Petri dishes inhibiting cellular attachment to the dish bottom. Any attachment of pSSCs to the Petri dish bottom was not observed by day 6 of culture (data not shown). Moreover, as shown in Figure 1, pSSCs plated on Petri dishes in GDNF-mESCCM and pSSCCM caused a significant decrease in pSSCs with AP activity from the beginning of culture (Figure 1), whereas GDNFmESCCM resulted in a significantly higher percentage of pSSCs (stained positively by AP staining) than pSSCCM throughout the entire culture period. These results demonstrate that a culture system consisting of a Petri dish and GDNF-mESCCM can be effectively used as an extracellular signaling-free microenvironment against pSSCs.

\section{Influence of different ECM protein-derived signals on pSSC AP activity}

Based on the developed extracellular signaling-free microenvironment, the effects of laminin- and collagen IVderived signaling on AP activity of pSSCs were investigated. Compared to pSSCs isolated from testis (day 0 in culture), non- (Figure 2A) or laminin-derived signals (Figure 2B) caused a significant decrease in the percentage of pSSCs

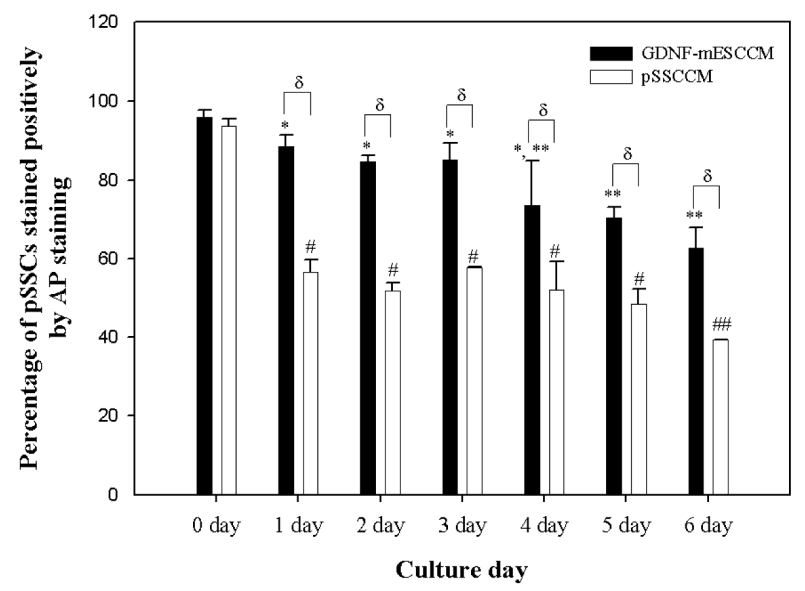

Figure 1. Determination of culture medium supporting the maintenance of AP activity in pSSC suspension culture. A suspension culture of $8 \times 10^{5}$ pSSCs was grown for 6 days on 35$\mathrm{mm}$ Petri dishes in GDNF-mESCCM or pSSCCM, and AP activity was analyzed daily. As a result, throughout the entire culture period, pSSCs cultured in GDNF-mESCCM showed significantly higher maintenance of AP activity than those in pSSCCM. Error bars represent standard deviations (SD). $\mathrm{N}=3 .{ }^{*}, * * \mathrm{p}<0.0001$ versus culture in GDNF-mESCCM. ${ }^{\#, \#} \mathrm{p}<0.0001$ versus culture in $\mathrm{pSSCCM} .{ }^{\delta} \mathrm{p}<0.0001$ between GDNF-mESCCM and pSSCCM on the same day of culture. AP, alkaline phosphatase; pSSCs, porcine spermatogonial stem cells; GDNF-mESCCM, glial cellderived neurotrophic factor-supplemented mouse embryonic stem cell culture medium; pSSCCM, porcine spermatogonial stem cell culture medium.

with AP activity on day 5 in culture and did not induce any significant difference in the maintenance of AP activity at day 3 of culture. In the case of collagen IV-derived signaling (Figure 2C), a significant decrease in AP activity was observed from day 3 in culture, while AP activity of pSSCs cultured at day 1 showed no significant difference compared to those pSSCs isolated from testis (day 0 of culture). Moreover, the lowest yield of pSSCs with AP
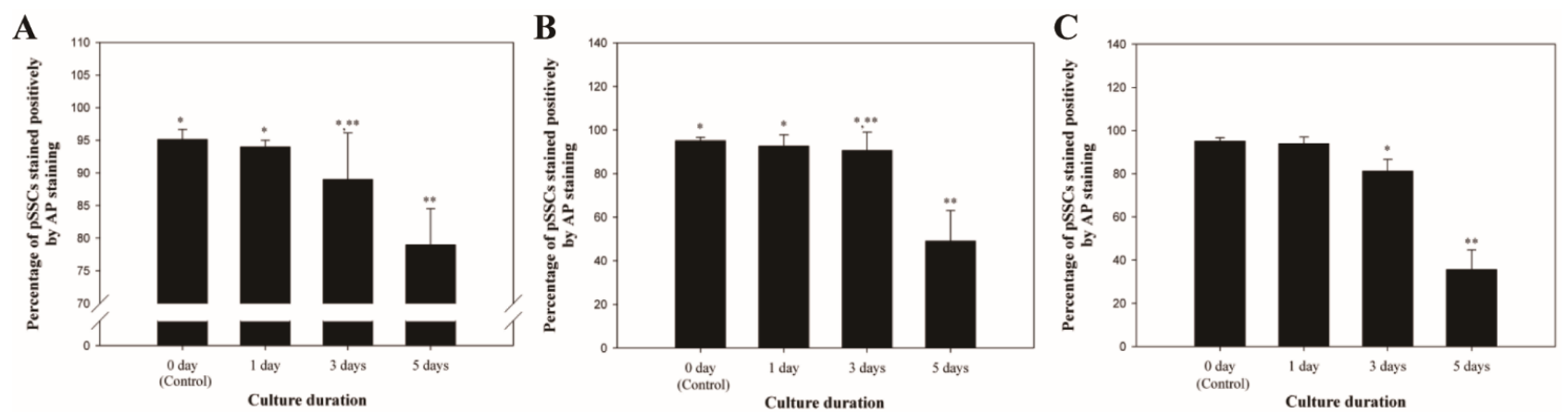

Figure 2. Effects of ECM protein-derived signals on the maintenance of AP activity in pSSCs. Cells $\left(8 \times 10^{5}\right.$ pSSCs $)$ were seeded onto non-, laminin- and collagen IV-coated Petri dishes and incubated in GDNF-mESCCM for 5 days. Subsequently, AP activity was analyzed at $0,1,3$, and 5 days of culture. Porcine SSCs cultured on non- (A) and laminin-coated Petri dishes (B) showed a significant decrease in AP activity after 5 days of culture. However, a significant decrease in AP activity post-3 days of culture was detected in pSSCs cultured on collagen IV-coated Petri dishes (C). Error bars represent standard deviations (SD). $\mathrm{N}=3 . *, * * \mathrm{p}<0.05$. ECM, extracellular matrix; AP, alkaline phosphatase; pSSCs, porcine spermatogonial stem cells; GDNF-mESCCM, glial cell-derived neurotrophic factor-supplemented mouse embryonic stem cell culture medium. 
activity was detected on day 5 of culture. Accordingly, we suggest that ECM protein-derived signaling plays an important role in regulating AP activity of pSSCs, and laminin-derived signaling is more effective at maintaining pSSC AP activity than collagen IV-derived signaling.

\section{Influence of different ECM protein-derived signals on transcriptional regulation of pSSC-specific gene expression}

Subsequently, ECM protein-derived signaling effects on the transcriptional regulation of SSC-specific genes were analyzed in unstimulated pSSCs or those stimulated with laminin or collagen IV. Moreover, the type of ECM proteinderived signaling supporting the maintenance of SSCspecific gene expression was also identified. As shown in Figure 3, laminin-derived signaling induced no significant transcriptional down-regulation of octamer-binding transcription factor 4 (OCT4), homeobox transcription factor nanog $(N A N O G)$, epithelial cellular adhesion molecule (EPCAM), THY-1 T-cell antigen (THY1), or ubiquitin carboxyl-terminal esterase L1 (UCHL1) throughout the entire culture period. However, pSSCs experiencing collagen IV-derived signaling showed significant down-regulation of EPCAM transcription post-1 day of stimulation and $U C H L 1$ transcription post-3 days of stimulation. Furthermore, significant transcriptional downregulation of EPCAM was detected in pSSCs cultured for 5 days in the absence of ECM protein-derived signaling. Moreover, upon comparison of the transcriptional level of SSC-specific genes in unstimulated pSSCs with those experiencing laminin- or collagen IV-derived signaling for the same time period, no significant differences in the transcriptional level of any gene expressed specifically in pSSCs was observed among any experimental group at 1 day of stimulation (Figure 4A). However, at 3 day of stimulation (Figure 4B), a significant transcriptional upregulation of EPCAM was detected in pSSCs experiencing laminin-derived signaling, which also showed the highest average transcription levels of OCT4, NANOG, THY1, and $U C H L 1$, although there were no significant differences among experimental groups. Simultaneously, collagen IVderived signaling showed no significant difference in SSCspecific gene transcription compared to the unstimulated signaling group. Finally, at 5 day of stimulation (Figure 4C),
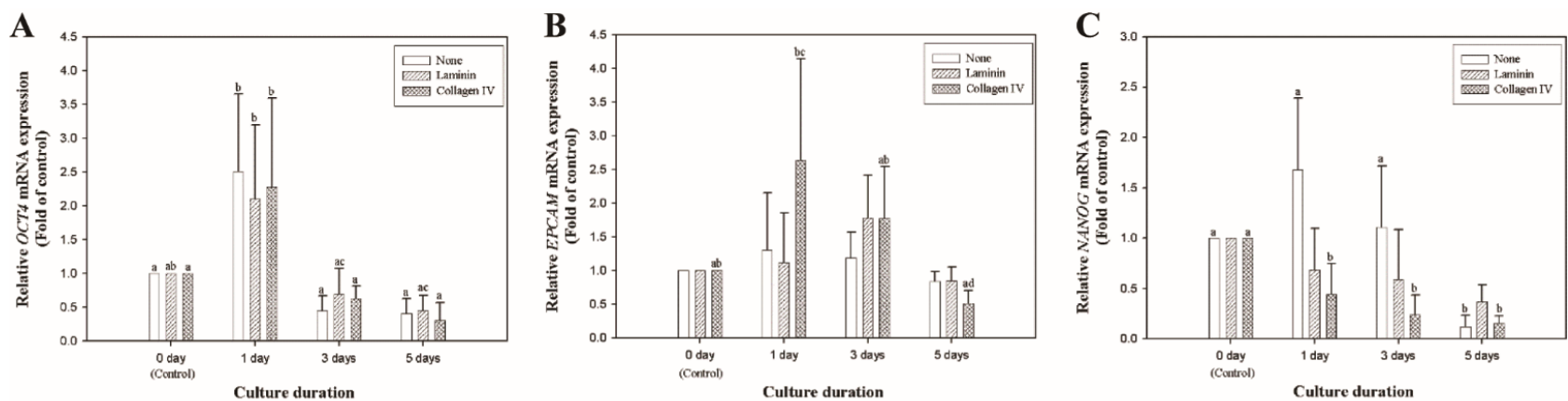


Figure 3. Effects of ECM protein-derived signals on the maintenance of transcriptional levels of SSC-specific genes in pSSCs. Cells $\left(8 \times 10^{5}\right.$ pSSCs) plated on non (None)-, laminin (Laminin)-, and collagen IV (Collagen IV)-coated 35-mm Petri dishes were cultured for 5 days in GDNFmESCCM. Transcriptional levels of SSC-specific genes in pSSCs cultured in each ECM protein for $0,1,3$, and 5 days were analyzed using realtime PCR. Despite culture duration, pSSCs stimulated by laminin-derived signals showed no significant decrease in the expression of any SSCspecific gene (OCT4, NANOG, EPCAM, THY1, or UCHL1). On the other hand, significant transcriptional down-regulation of EPCAM (C) was detected in pSSCs cultured for 5 days on non- and collagen IV-coated culture plates, and a significant decrease in UCHL1 (E) was observed in pSSCs experiencing collagen IV-derived signaling for 3 and 5 days. Error bars represent standard deviations (SD) $n=3$. ${ }^{\text {ab }} p<0.05$ versus culture duration in the 'None' group. ${ }^{\mathrm{a}-\mathrm{c}} \mathrm{p}<0.05$ versus culture duration in the 'Laminin' group. ${ }^{\mathrm{a}-\mathrm{d}} \mathrm{p}<0.05$ versus culture duration in the 'Collagen IV' group. ECM, extracellular matrix; SSC, spermatogonial stem cell; pSSCs, porcine spermatogonial stem cells; GDNF-mESCCM, glial cellderived neurotrophic factor-supplemented mouse embryonic stem cell culture medium; PCR, polymerase chain reaction; OCT4, octamer-binding transcription factor 4; NANOG, homeobox transcription factor nanog; EPCAM, epithelial cellular adhesion molecule; THY1, THY-1 T-cell antigen; UCHL1, ubiquitin carboxyl-terminal esterase L1. 

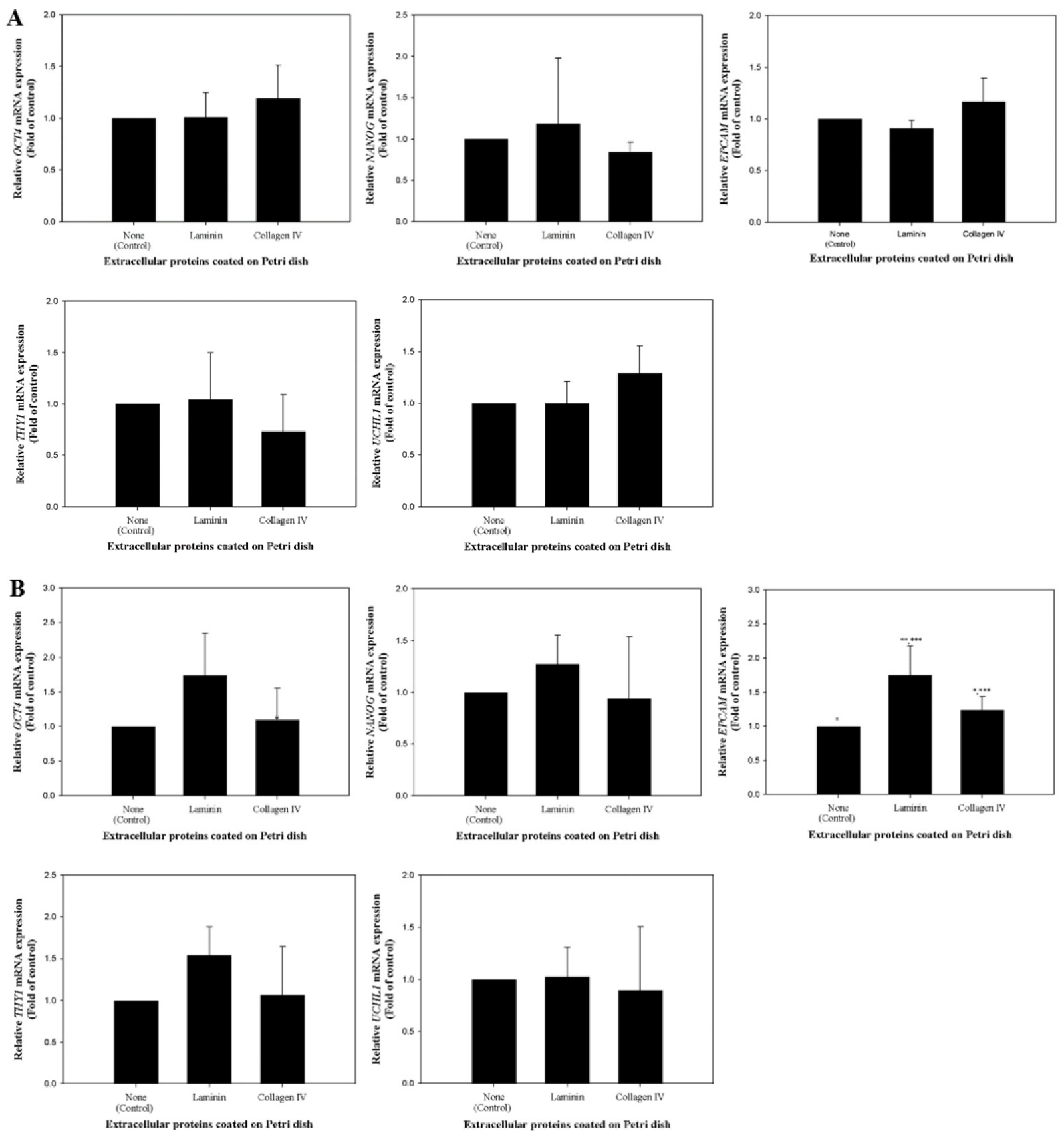

Figure 4. i) Comparison of SSC-specific gene transcription levels in pSSCs subjected to the indicated ECM protein-derived signals for 1, 3, and 5 days. Cells $\left(8 \times 10^{5} \mathrm{pSSCs}\right)$ were plated onto non-, laminin- and collagen IV-coated Petri dishes and incubated for 5 days in GDNF-mESCCM. Subsequently, transcription levels of SSC-specific genes were estimated at day 1, 3, and 5 of culture using real-time PCR, respectively. In the culture for 1 day (A), no significant alterations in the transcriptional regulation of OCT4, NANOG, EPCAM, THY1, or UCHL1 was induced by laminin- or collagen IV-derived signaling. In case of pSSCs cultured for 3 days (B), pSSCs experiencing laminin-derived signaling showed the highest transcriptional level of EPCAM and the highest average transcription levels of OCT4, NANOG, THY1, and UCHL1, although the differences were not significant. On the other hand, collagen IV-derived signaling in pSSCs showed no significant effects on the transcriptional regulation of SSC-specific genes. Moreover, in case of pSSCs cultured for 5 days (C), although no significant decrease in OCT4, EPCAM, THY1 or $U C H L 1$ expression was observed, a significant increase in $N A N O G$ expression was revealed in pSSCs experiencing laminin-derived signaling. In contrast, collagen IV-derived signaling induced significant transcriptional down-regulation of THY1. Error bars represent standard deviations (SD). $\mathrm{n}=3$. $^{* * * * *} \mathrm{p}<0.05$. SSC, spermatogonial stem cell; pSSCs, porcine spermatogonial stem cells; ECM, extracellular matrix; GDNFmESCCM, glial cell-derived neurotrophic factor-supplemented mouse embryonic stem cell culture medium; PCR, polymerase chain reaction; $O C T 4$, octamer-binding transcription factor 4; NANOG, homeobox transcription factor nanog; EPCAM, epithelial cellular adhesion molecule; THY1, THY-1 T-cell antigen; UCHL1, ubiquitin carboxyl-terminal esterase L1. 

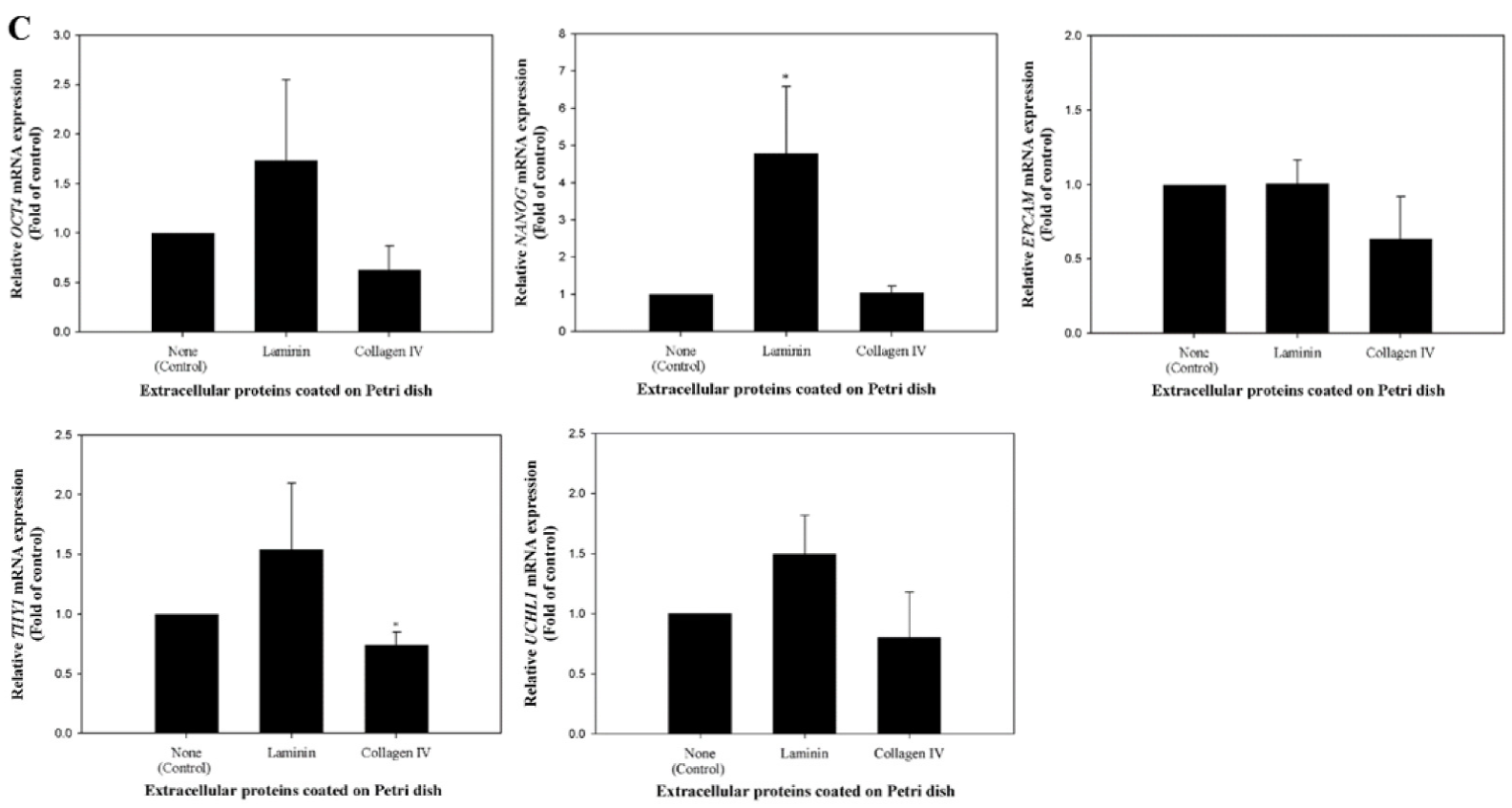

Figure 4. ii) Comparison of SSC-specific gene transcription levels in pSSCs subjected to the indicated ECM protein-derived signals for 1, 3, and 5 days. Cells $\left(8 \times 10^{5} \mathrm{pSSCs}\right)$ were plated onto non-, laminin- and collagen IV-coated Petri dishes and incubated for 5 days in GDNF-mESCCM. Subsequently, transcription levels of SSC-specific genes were estimated at day 1, 3, and 5 of culture using real-time PCR, respectively. In the culture for 1 day (A), no significant alterations in the transcriptional regulation of OCT4, NANOG, EPCAM, THY1, or UCHL1 was induced by laminin- or collagen IV-derived signaling. In case of pSSCs cultured for 3 days (B), pSSCs experiencing laminin-derived signaling showed the highest transcriptional level of EPCAM and the highest average transcription levels of OCT4, NANOG, THY1, and UCHL1, although the differences were not significant. On the other hand, collagen IV-derived signaling in pSSCs showed no significant effects on the transcriptional regulation of SSC-specific genes. Moreover, in case of pSSCs cultured for 5 days (C), although no significant decrease in OCT4, EPCAM, THY1 or UCHL1 expression was observed, a significant increase in $N A N O G$ expression was revealed in pSSCs experiencing laminin-derived signaling. In contrast, collagen IV-derived signaling induced significant transcriptional down-regulation of THY1. Error bars represent standard deviations (SD). $\mathrm{n}=3{ }^{*-* * *} \mathrm{p}<0.05$. SSC, spermatogonial stem cell; pSSCs, porcine spermatogonial stem cells; ECM, extracellular matrix; GDNFmESCCM, glial cell-derived neurotrophic factor-supplemented mouse embryonic stem cell culture medium; PCR, polymerase chain reaction; OCT4, octamer-binding transcription factor 4; $N A N O G$, homeobox transcription factor nanog; EPCAM, epithelial cellular adhesion molecule; THY1, THY-1 T-cell antigen; UCHL1, ubiquitin carboxyl-terminal esterase L1.

significant transcriptional up-regulation of $N A N O G$ transcription levels was induced by laminin-derived signaling, but no significant decrease in OCT4, EPCAM, THY1, or UCHL1 was observed. In contrast, collagen IVderived signaling showed significant transcriptional downregulation of THY1 but no significant increase in OCT4, NANOG, EPCAM, or UCHL1 levels. These results demonstrate that laminin-derived signaling is effective in terms of sustaining the transcriptional level of genes transcribed specifically in pSSCs without any transcriptional down-regulation.

\section{DISCUSSION}

In this study, the culture of pSSCs on laminin-coated Petri dishes did not induce any significant decrease in AP activity by day 3 of culture or the transcription of SSCspecific genes throughout the entire culture duration (5 days), whereas collagen IV-derived signaling showed maintenance in AP activity and SSC-specific gene transcription by day 1 of culture. Furthermore, pSSCs cultured on uncoated Petri dishes showed no significant decrease in AP activity by day 3 of culture and maintained transcriptional levels of all genes transcribed specifically in pSSCs by day 3 of culture. Accordingly, these results demonstrate that laminin, among the diverse ECM proteins comprising the STBM, is a key protein involved in maintaining the undifferentiated state of SSCs in porcine testis.

As shown in Figure 1, GDNF-mESCCM showed stronger maintenance of pSSC undifferentiation on the Petri dishes coated without any ECM proteins than pSSCCM, indicating that, under extracellular signal-free niche, physico-chemical and physiological niche of GDNFmESCCM are appropriate further for maintaining undifferentiation of pSSCs. Especially, as factors resulting in stimulating loss of AP activity in the pSSCs, bFGF, and EGF included in pSSCCM can be considered, supported by the previous reports that bFGF and/or EGF induce differentiation of stem cells in the extracellular signal-free niche (Carpenter and Cohen, 1990; Schuldiner et al., 2000; Del Angel-Mosqueda et al., 2015). Accordingly, physico- 
chemical and physiological niche derived from GDNFmESCCM will be able to be used as a basic microenvironment for investigating effects of extracellular signals on undifferentiation or differentiation of pSSCs.

Porcine SSCs cultured on laminin-coated Petri dishes showed a similar tendency in the maintenance of AP activity as those on uncoated Petri dishes (Figure 2) and no significant down-regulation of any SSC-specific gene transcription, regardless of culture period, compared to pSSCs isolated from the testis with no in vitro culturing (Figure 3). Specifically, they showed the highest transcriptional level of all SSC-specific genes at day 5 of culture (Figure 4C) among all experimental groups. These results indicate that laminin-derived signaling does not affect AP activity in pSSCs, but greatly contributes to sustaining the undifferentiated state.

However, the exposure of collagen IV-derived signaling to pSSCs caused a marked decrease in AP activity and significant down-regulation of SSC-specific gene transcription (Figure 2, 3, and 4). These results suggest that collagen IV-derived signaling more strongly stimulates the differentiation of pSSCs than maintaining undifferentiated pSSCs, which is supported by previous studies describing how collagen made it difficult to maintain the undifferentiated state of stem cells (Shinohara et al., 1999; McLean, 2008). Therefore, collagen IV may represent a key factor inducing guided differentiation of pSSCs into sperms.

In conclusion, we identified the importance of lamininderived signaling in the maintenance of pSSC undifferentiation. Although laminin had no significant effect on the inhibition of pSSC differentiation, the combination of laminin with other ECM proteins may synergistically result in both the maintenance of pSSC undifferentiation and inhibition of pSSC differentiation. Accordingly, additional studies will be required to substantiate our findings. Moreover, these results will contribute to the development of a cellular niche supporting self-renewal of pSSCs and stimulating pSSC differentiation into sperms.

\section{CONFLICT OF INTEREST}

We certify that there is no conflict of interest with any financial organization regarding the material discussed in the manuscript.

\section{ACKNOWLEDGMENTS}

This research was supported by Korea Institute of Planning and Evaluation for Technology in Food, Agriculture, Forestry and Fisheries (IPET) through (AgriBioindustry Technology Development Program), Funded by Ministry of Agriculture, Food and Rural Affairs (MAFRA) (IPET112015-4)

\section{REFERENCES}

Aponte, P. M., M. P. van Bragt, D. G. de Rooij, and A. M. van Pelt. 2005. Spermatogonial stem cells: Characteristics and experimental possibilities. APMIS 113:727-742.

Aumailley, M. and R. Timpl. 1986. Attachment of cells to basement membrane collagen type IV. J. Cell Biol. 103:15691575.

Brinster, R. L. 2002. Germline stem cell transplantation and transgenesis. Science 296:2174-2176.

Carpenter, G. and S. Cohen. 1990. Epidermal growth factor. J. Biol. Chem. 265:7709-7712.

Chen, S., M. Lewallen, and T. Xie. 2013. Adhesion in the stem cell niche: biological roles and regulation. Development 140:255265

Daley, G. Q. and D. T. Scadden. 2008. Prospects for stem cellbased therapy. Cell 132:544-548.

de Barros, F. R. O., M. I. Giassetti, and J. A. Visintin. 2012. Spermatogonial stem cells and animal transgenesis In: Innovations in Biotechnology (Ed. Eddy C. Agbo). InTech, Rijeka, Croatia. pp. 303-318.

de Rooij, D. G. and J. A. Grootegoed. 1998. Spermatogonial stem cells. Curr. Opin. Cell Biol. 10:694-701.

Del Angel-Mosqueda, C., Y. Gutiérrez-Puente, A. P. López-Lozano, R. E. Romero-Zavaleta, A. Mendiola-Jiménez, C. E. MedinaDe la Garza, M. Márquez-M, and M. A. De la Garza-Ramos. 2015. Epidermal growth factor enhances osteogenic differentiation of dental pulp stem cells in vitro. Head Face Med. 11:29.

Gattazzo, F., A. Urciuolo, and P. Bonaldo. 2014. Extracellular matrix: A dynamic microenviroment for stem cell niche. Biochim. Biophys. Acta 1840:2506-2519.

Guan, K., K. Nayernia, L. S. Maier, S. Wagner, R. Dressel, J. H. Lee, J. Nolte, F. Wolf, M. Li, W. Engel, and G. Hasenfuss. 2006. Pluripotency of spermatogonial stem cells from adult mouse testis. Nature 440:1199-1203.

Hofmann, M. C. 2008. Gdnf signaling pathways within the mammalian spermatogonial stem cell niche. Mol. Cell. Endocrinol. 288:95-103.

Kanatsu-Shinohara, M., J. Lee, K. Inoue, N. Ogonuki, H. Miki, S. Toyokuni, M. Ikawa, T. Nakamura, A. Ogura, and T. Shinohara. 2008. Pluripotency of a single spermatogonial stem cell in mice. Biol. Reprod. 78:681-687.

Kanatsu-Shinohara, M. and T. Shinohara. 2013. Spermatogonial stem cell self-renewal and development. Annu. Rev. Cell Dev. Biol. 29:163-187.

Khoshnoodi, J., V. Pedchenko, and B. G. Hudson. 2008. Mammalian collagen IV. Microsc. Res. Tech. 71:357-370.

Kubota, H. and R. L. Brinster. 2006. Reproductive endocrinology (including placental hormones): Technology Insight: In vitro culture of spermatogonial stem cells and their potential therapeutic uses. Nat. Rev. Endocrinol. 2:99-108.

Lavitrano, M., M. Busnelli, M. G. Cerrito, R. Giovannoni, S. Manzini, and A. Vargiolu. 2005. Sperm-mediated gene transfer. Reprod. Fertil. Dev. 18:19-23.

Lee, W. Y., H. J. Park, R. Lee, K. H. Lee, Y. H. Kim, B. Y. Ryu, N. H. Kim, J. H. Kim, J. H. Kim, S. H. Moon, J. K. Park, H. J. Chung, D. H. Kim, and H. Song. 2013. Establishment and in vitro culture of porcine spermatogonial germ cells in low 
temperature culture conditions. Stem Cell Res. 11:1234-1249.

Lim, J. J., H. J. Kim, K. S. Kim, J. Y. Hong, and D. R. Lee. 2013. In vitro culture-induced pluripotency of human spermatogonial stem cells. Biomed. Res. Int. 2013:143028.

Luo, J., S. Megee, and I. Dobrinski. 2009. Asymmetric distribution of UCH-L1 in spermatogonia is associated with maintenance and differentiation of spermatogonial stem cells. J. Cell. Physiol. 220:460-468.

McLaren, A. 2003. Primordial germ cells in the mouse. Dev. Biol. 262:1-15.

McLean, D. J. 2008. Spermatogonial stem cell transplantation, testicular function, and restoration of male fertility in mice. Methods Mol. Biol. 450:149-162.

Nagano, M., C. J. Brinster, K. E. Orwig, B. Y. Ryu, M. R. Avarbock, and R. L. Brinster. 2001. Transgenic mice produced by retroviral transduction of male germ-line stem cells. Proc. Natl. Acad. Sci. USA. 98:13090-13095.

Oatley, J. M. and R. L. Brinster. 2006. Spermatogonial stem cells. Methods Enzymol. 419:259-282.

Oatley, J. M. and R. L. Brinster. 2008. Regulation of spermatogonial stem cell self-renewal in mammals. Annu. Rev. Cell Dev. Biol. 24:263-286.

Öhlund, D., O. Franklin, E. Lundberg, C. Lundin, and M. Sund. 2013. Type IV collagen stimulates pancreatic cancer cell proliferation, migration, and inhibits apoptosis through an autocrine loop. BMC Cancer 13:154.

Ooba, T., T. Ishikawa, K. Yamaguchi, Y. Kondo, Y. Sakamoto, and M. Fujisawa. 2008. Expression and distribution of laminin chains in the testis for patients with azzospermia. J. Androl. 29:147-152.

Park, M. H., J. E. Park, M. S. Kim, K. Y. Lee, H. J. Park, J. I. Yun, J. H. Choi, E. S. Lee, and S. T. Lee. 2014. Development of a high-yield technique to isolate spermatogonial stem cells from porcine testes. J. Assist. Reprod. Genet. 31:983-991.
Phillips, B. T., K. Gassei, and K. E. Orwig. 2010. Spermatogonial stem cell regulation and spermatogenesis. Philos. Trans. R. Soc. Lond., B, Biol. Sci. 365:1663-1678.

Pöschl, E., U. Schlötzer-Schrehardt, B. Brachvogel, K. Saito, Y. Ninomiya, and U. Mayer. 2004. Collagen IV is essential for basement membrane stability but dispensable for initiation of its assembly during early development. Development 131:1619-1628.

Schuldiner, M., O. Yanuka, J. Itskovitz-Eldor, D. A. Melton, and N Benvenisty. 2000. Effects of eight growth factors on the differentiation of cells derived from human embryonic stem cells. Proc. Natl. Acad. Sci. USA. 97:11307-11312.

Shinohara, T., M. R. Avarbock, and R. L. Brinster. 1999. Beta1and alpha6-integrin are surface markers on mouse spermatogonial stem cells. Proc. Natl. Acad. Sci. USA. 96:5504-5509.

Simon, L., R. A. Hess, and P. S. Cooke. 2010. Spermatogonial stem cells, in vivo transdifferentiation and human regenerative medicine. Expert Opin. Biol. Ther. 10:519-530.

Siu, M. K. and C. Y. Cheng. 2004. Extracellular matrix: recent advances on its role in junction dynamics in the seminiferous epithelium during spermatogenesis. Biol. Reprod. 71:375-391.

Siu, M. K. and C. Y. Cheng. 2008. Extracellular matrix and its role in spermatogenesis. Adv. Exp. Med. Biol. 636:74-91.

Timpl, R., H. Rohde, P. G. Robey, S. I. Rennard, J. M. Foidart, and G. R. Martin. 1979. Laminin-a glycoprotein from basement membranes. J. Biol. Chem. 254:9933-9937.

Vlajković, S., R. Cukuranović, M. D. Bjelaković, and V. Stefanović. 2012. Possible therapeutic use of spermatogonial stem cells in the treatment of male infertility: A brief overview. Sci. World J. 2012:Article ID 374151.

Yazama, F., M. Esaki, and H. Sawada. 1997. Immunocytochemistry of extracellular matrix components in the rat seminiferous tubule: Electron microscopic localization with improved methodology. Anat. Rec. 248:51-62. 